\title{
Potentialities of Sentinel-1 for mapping and monitoring geological and cryospheric processes in the Patagonia region (Chile)
}

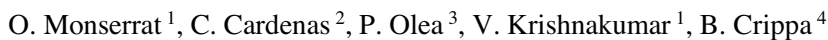 \\ ${ }^{1}$ Centre Tecnològic de Telecomunicacions de Catalaunya, Av. Carl Friedrich Gauss, 4, Castelldefels, Spain, omonserrat@cttc.cat \\ and vkrishnakumar@cttc.cat \\ ${ }^{2}$ Gaia -Antarctic Research Center, Universidad de Magallanes, carlos.cardenas@umag.cl \\ ${ }^{3}$ servicio Nacional de Geología y Minería, Avda. Santa María 0104, Santiago, Chile. \\ ${ }^{4}$ Department of Earth Sciences, University of Milan, Milan, Italy
}

KEY WORDS: Active geohazards, SAR interferometry, Sentinel-1, Glacier monitoring

\begin{abstract}
:
This work shows two examples on the use of Sentinel-1 data for monitoring different natural processes, like active geohazards or glacier dynamics in the Patagonia region. Sentinel-1 is a two-satellite constellation, launched by the European Space Agency (ESA), that provides SAR imagery with interferometric capabilities. It is in operation since 2014 and has supposed a significant improvement in the exploitation of these type of data for applications like natural hazards mapping and monitoring. The acquisition policy, that guarantees an acquisition each few days (12 days in Patagonia region) for both ascending and descending trajectories, and the data distribution policy, that allows free access to the images without legal constrains, are the main reasons for this improvement. These two aspects allowed not only to assure the data in the past but also to perform monitoring plans at medium-long term. In this work we show the potentialities of the use of these data in the Patagonia region through the application of two different techniques in two
\end{abstract} different application test sites: urban areas and glaciers.

\section{INTRODUCTION}

Since their appearance in the 80s, satellite Synthetic Aperture Radar (SAR) systems have been widely used for the study of natural processes like volcanic activity (Hooper et al. 2004, Antonielli et al. 2014), landslides (Bovenga et al. 2012, Herrera et al. 2009), subsidences (Amelung et al. 1999, Biescas et al. 2007) and glacier dynamics (Strozzi et al. 2002, Burgess et al. 2013) or snow cover (Pettinato et al. 2012). The ability to observe independently of light and weather conditions together with a relatively high spatial resolution makes SAR sensor a reliable tool for these type of applications.

The SAR data can be decomposed into two main components: the phase and the amplitude (Ferretti et al. 2007). The phase contains the information related to the sensor-target distance. It can be exploited by analyzing the phase changes between images acquired from different points of view or at different times. The technique that exploits these differences is named SAR interferometry (InSAR) and has been widely used for digital elevation model generation (Van Zyl 2001, Krieger et al. 2007) or terrain movement measurement and monitoring (Crosetto et al. 2016). It allows the detection and monitoring of slow ground movements, up to millimeter order. The main reason is because it measures changes comparable to the wavelength. The main applications have been related to different geological processes such as landslides, subsidence's or volcanic activity or earthquakes (Notti et al. 2014, Osmanoğlu et al. 2011, Lundgren et al. 2004, Massonnet and Feigl 1998).

The amplitude component measures how strong the sensor receives the echo of the illuminated target. It mainly depends on the physical characteristics of the target like orientation or rugosity (Ferretti et al. 2007). The amplitude data has been applied for classical fields of remote sensing like land cover mapping (Waske et al. 2009), soil moisture (Moran et al. 2000), flood mapping (Brivio et al. 2002), archaeology (Conesa et al. 2014) but also for fields like landslide mapping (Mondini et al. 2019). Several works in the literature show applications of the amplitude to measure displacements through offset tracking techniques (Singleton et al. 2014). The application of offset tracking allows to perform both quantitative and qualitative analysis of the dynamics of different objects such as glaciers (Strozzi et al. 2002) or landslides (Singleton et al. 2014).

Despite the high potentialities of SAR data for geohazard and glacier monitoring, its use has been mainly limited to scientific purposes. This has been mainly due to the lack of acquisition plans that resulted in a limited coverage with enough temporal sampling. This made difficult the development of operational applications like long term monitoring plans. Moreover, the distribution policies, which usually has been accompanied by a commercial exploitation of the images, has also hindered its application when compared with more consolidated techniques like GPS. This has significantly changed with the appearance on stage of the Sentinel-1 (A and B) constellation of the European space agency (ESA) (Torres et al. 2012). Sentinel-1 has changed both the acquisition plan and the distribution policy. The guarantee of a worldwide coverage with a revisiting time between 6 and 24 days ( 12 days in Patagonia) and the free access to the data allowed the generation of operational tools and plans for relatively mapping and monitoring natural processes (Gonzalez et al. 2015, Barra et al. 2016, Barra et al. 2017, Raspini et al. 2018). This work aims to show the potentialities of Sentinel1 for mapping and monitoring natural processes in Patagonia region. This is shown by describing the results obtained over two areas of the region: The Punta Arenas city where the ground displacements are measured; and the Shoutern Patagonia Icefield site where the displacements of three glaciers are measured.

The paper is organized in two main sections that briefly address the used data, the methodology and the results of each test site. Then it closes with some conclusions.

\section{SENTINEL-1 SAR INTERFEROMETRY FOR MAPPING AND MONITORING SLOW MOVEMENTS: THE PUNTA ARENAS TEST SITE}

In the last decades Differential SAR interferometry (DInSAR) has been widely used for ground displacement measurement. The displacements are measured by exploiting the phase differences between SAR images acquired on the same area at different 
times. The interferometric phase is characterized by the following equation:

$$
\Delta \varphi=\varphi_{\text {disp }}+\varphi_{\text {topo }}+\varphi_{\text {APS }}+\varphi_{\text {noise }}+2 * k * \pi
$$

where $\Delta \varphi$ is the interferometric phase (observation), $\varphi_{\text {disp }}$ is the contribution of the displacement to the phase, $\varphi_{\text {topo }}$ is the contribution of the terrain topography to the phase. It is due to the different points of view of the satellite at the two acquisition times. Its main contribution is removed by using a DTM of the measured area. However, it is a remaining component due to the DTM errors $\varphi_{\text {etopo }}$ that must be estimated. $\varphi_{\text {noise }}$ is the phase change due to physical changes of the measured object. Finally the term $2 * k * \pi$ is related to the number of wave cycles that the emitted signal does in the sensor-target-sensor path. The estimation of the $k$ is done relatively to a given reference. The estimation process is named phase unwrapping. The estimation of displacements consists in discriminating the displacement component from the other together with the estimation of the $k$ number (Devanthery et al. 2014).

The $\varphi_{\text {disp }}$ can be sometimes directly derived from a single interferogram (Massonet and Feigl 1998). However, this only happens when the displacement component is at least one order of magnitude bigger than the others. When this not happens, it is needed the use of stack of interferograms, estimation methods and spatio-temporal filters to properly discriminate the displacement component from the others. In the literature can be found several approaches that deals with this problem (Ferretti et al. 2000, Lanari et al. 2004,). Crosetto et al. 2016 provides an extensive review of the existing approaches.

From the technical point of view, Sentinel-1 has supposed a step forward in the displacement estimation capabilities. The fact of having images each 6-12 days has provided a significant reduction of the influence of $\varphi_{\text {noise }}$ in some areas where it was not possible to measure with the "old" SAR satellites. Moreover, the combination of short temporal baselines and medium size wavelength $(5.66 \mathrm{~cm})$ allowed the detection and measurement of relatively fast displacements with interesting applications to landslide mapping (Barra et al. 2016 and Raspini et al 2018) or glacier inventorying (Villarroel et al. 2018). Figure 1 shows an example of amplitude image (A) and a 12 days interferogram (B) over the area of Punta Arenas. It can be observed the low level of noise in the phase including natural areas.

\subsection{Dataset and used approach}

In this work we applied an approach based on the PSIG technique (Devanthéry et al. 2014) to process the metropolitan area of Punta Arenas (red rectangle in Figure 1 A). For this purpose, 89 IWSLC Sentinel-1 images acquired in ascending trajectory have been used. The covered period ranges from December 2014 to July 2019. The procedure is described here below:

Selection of Persistent Scatterers (PS) candidates: The PS candidates has been selected by using the dispersion of amplitude (DA) (Ferretti et al. 2007). The DA threshold is 0.3 . The threshold was selected as a trade-off between phase noise and coverage. The number of PS-candidates is 63828 .

- PS height estimation: in this step is estimated the $\varphi_{\text {etopo }}$. The estimation method is the periodogram (Ferretti et al. 2000). The input data were 88 interferograms calculated from consecutive images (89). Only those point with temporal coherence higher than 0.5 were selected (PSs here in advance). Again the threshold responds to a trade-off between noise and coverage.
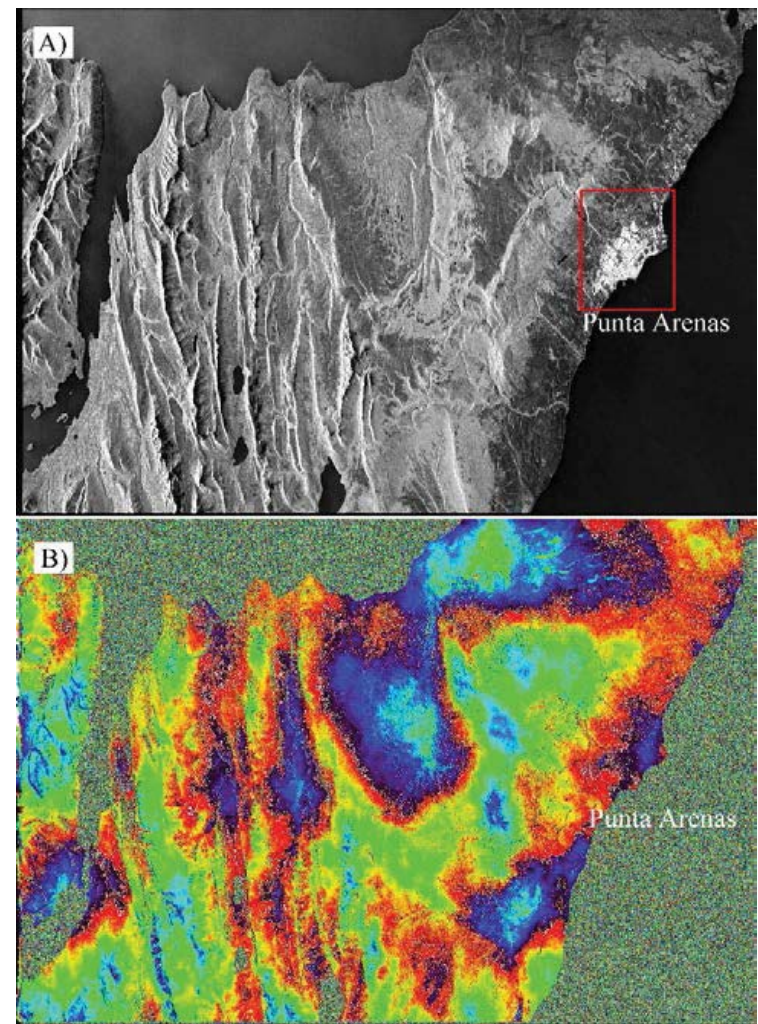

Figure 1. (A) Amplitude image of the Punta Arenas area. (B) 12-day interferogram.

- Removal of the PS Height: the estimated $\varphi_{\text {etopo }}$ is removed from the original interferograms. This step is done in order to minimize the effects of the topographic error, smoothing the interferometric phase and easing the phase unwrapping.

- Displacement time series estimation: this step includes the phase unwrapping and the atmospheric phase component removal (APS). The phase unwrapping is done by using the 2+1D phase unwrapping approach described in Devanthery et al. 2014. In this context, the use of Sentinel-1 allows to obtain redundant networks of interferograms with short temporal baselines. This helps to avoid phase unwrapping errors due to relatively fast displacements. Then after, the APS is removed using spatio-temporal filters (Ferretti et al. 2000). The result of this step is, for each selected point, the displacement time series in $\mathrm{mm}$.

Velocity estimation and geocoding: for each PS is estimated the displacement velocity in $\mathrm{mm} / \mathrm{yr}$ by using a robust linear regression and the time series. Finally, the PS is geocoded, i.e. its ground coordinates are obtained.

\subsection{Results}

Figure 2 shows the obtained displacement velocity map superimposed to a google image. The total number of measured points is 59418. The standard deviation, taking into account all the measured points, is $1.24 \mathrm{~mm} / \mathrm{yr}$. However, if we focus in the city centre we obtain a standard deviation of $0.7 \mathrm{~mm} / \mathrm{yr}$. The stability threshold has been fixed as $\pm 3 \mathrm{~mm} / \mathrm{yr}$ that is approximately 2.5 times the standard deviation. This is a standard threshold to avoid potential false deformation due to spurious points in wide areas (Barra et al. 2017). 97\% of the points are below the stability threshold, i.e. they are stable. 


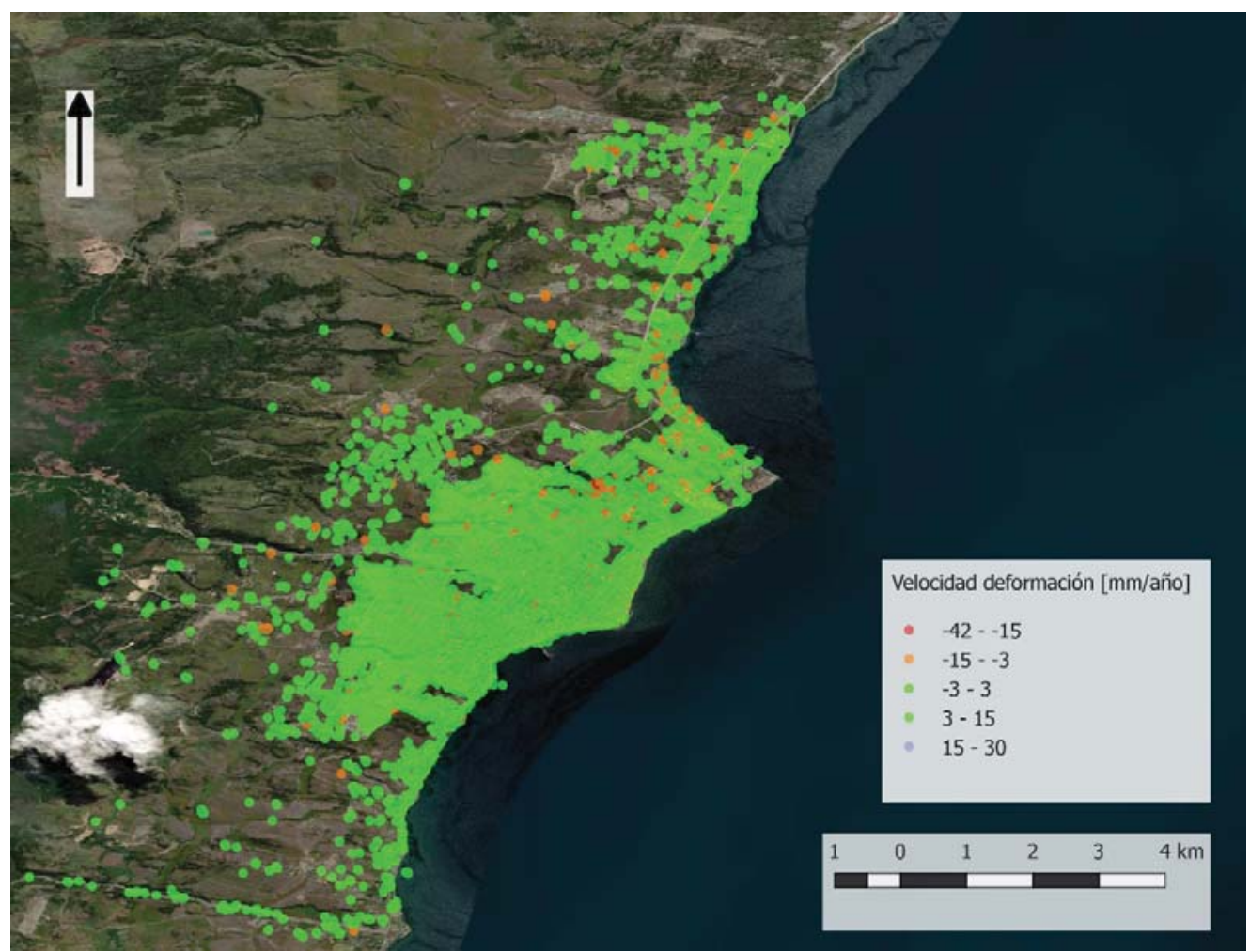

Figure 2. Displacement velocity map of Punta Arenas.

Some displacement areas can be observed in Figure 2. The maximum displacement velocity is $20 \mathrm{~mm} / \mathrm{yr}$ and is located in the square $\mathrm{A}$ of Figure 2. Figure 3 shows a zoom on this area. It corresponds to a residential area built up at the start of this century. Figure 3 shows also the displacement time series obtained as an average of the points located in the displacement area (orange) and of the ones framed in the figure and without movement (green). It can be seen that the movement is almost linear. It accumulates up to $90 \mathrm{~mm}$ of displacement. In this case, the movement can be explained by a settlement process.

A second example is shown in Figure 4. In this case again the movements could be explained by settlement process given that the area affected by displacements has grown in the last 10 years. It can be seen also that the temporal series are slightly noisier than in the Figure 3.

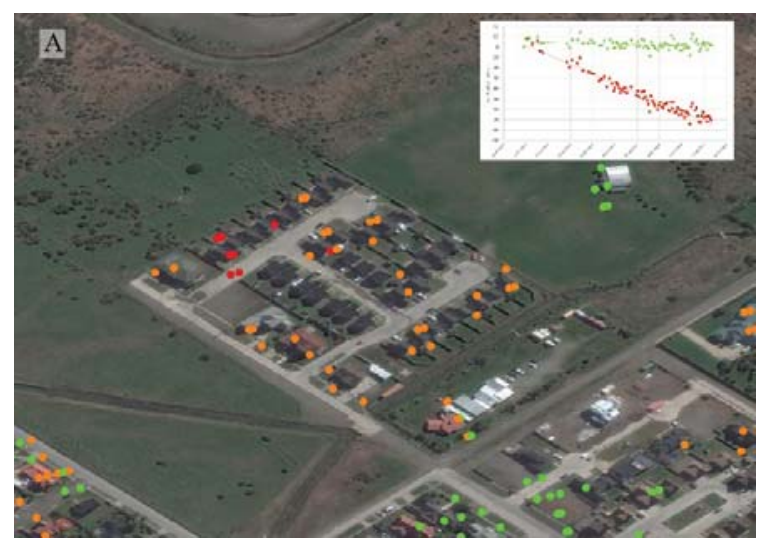

Figure 3. Example of displacement area and time series.

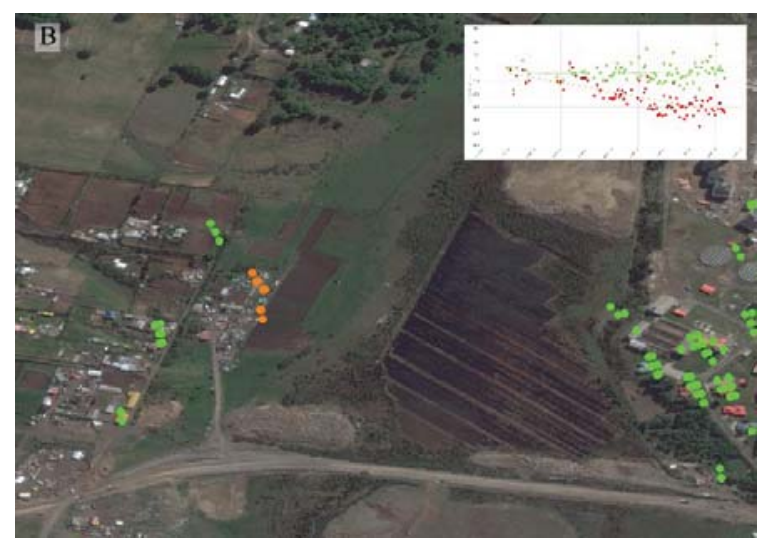

Figure 4. Example of displacement area and time series.

\section{SENTINEL-1 AMPLITUDE FOR MAPPING AND GLACIER DISPLACEMENTS}

This section shows the potentialities of exploiting the Sentinel-1 amplitude to map the displacements of glaciers in remote regions. The used technique is the offset-tracking. It is a well-known technique widely used for displacement measurement. Examples of application can be found in Strozzi et al. 2002 and Singleton et al. 2014. It consists on the exploitation of the cross-correlation between two SAR amplitude images (Ferretti et al. 2007) to estimate the displacements (Monserrat et al. 2013). It can be applied in two different ways: (i) by co-registering two images and obtaining the displacement information from the coregistration residuals or (ii) by applying the cross-correlation to already co-registered images in order to estimate the offsets in 


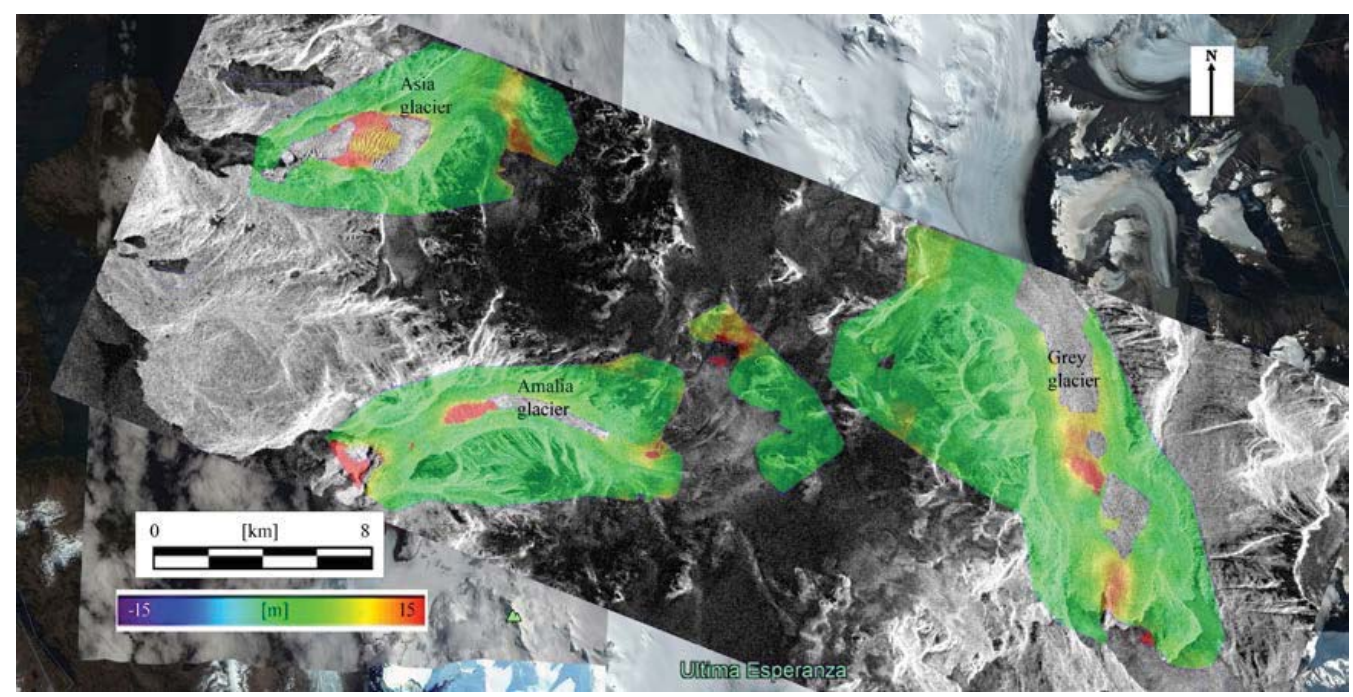

Figure 5. Displacement map superimposed to a geocoded amplitude and an optical image of Google Earth.

the displacement areas. In this work we use the second approach. The main steps are

\section{- Image downloading and precise orbit extraction.}

- Co-registration of the amplitude images: this step consists on resampling both images in the way of that the same pixel coordinates represent the same footprint on the floor. For this purpose, are used the precise orbits provided by ESA (aux.sentinel1.eo.esa.int). The alignment of the Sentinel-1 images is done using the position of the satellite and therefore, without the influence of movements. This is important when we work in local areas or areas where there are not stable points. Finally, it is worth noting that, in the case of Sentinel-1 data, the precise orbits are good enough to provide a sub-pixel level co-registration.

- Offset-estimation on the co-registered images: in this step we apply the cross-correlation on the already co-registered images. All the measured offsets with cross-correlation higher than a given threshold are considered movements. The crosscorrelation is estimated on a window (Monserrat et al. 2013). In this work the window size for cross-correlation estimation is 32 pixels and the selected oversampling is 64 .

\subsection{Dataset and Results}

We applied the approach to a pair of Sentinel-1 IW SLC images acquired the $14^{\text {th }}$ of January and the $7^{\text {th }}$ February of 2019. The selected area is located in the southern part of "Campo de Hielo Sur" and includes both the Amalia, Asia and Grey glaciers. Figure 5 shows the result obtained. The selected points have cross-correlation higher than 0.5 . The standard deviation of the whole dataset is $2.4 \mathrm{~m}$. This value can be set as sensitivity threshold. We can observe, in the three glaciers, displacements up to 15 meters in 24 days. This is equivalent to $0.63 \mathrm{md}^{-1}$. In particular, in the Grey glacier, this number is sensible smaller than the one measured for a similar period in 2015 and in the same area by Schwalbe et al. 2017 which was around $1.6 \mathrm{md}^{-1}$. Further analysis is required to interpret this change as something relevant or not. Figures 6 and 7 show a zoom over the Amalia glacier and the Asia glacier. The arrows show the direction of the displacements. What we can observe in both figures is that the main displacements are measured in those areas where can be observed geometrical features. This is one of the limitations of the approach, it requires of geometric information in the images to get good correlation and therefore to be capable to estimate the displacements. In any case, the obtained results are in agreement with the displacements measurable by eye inspection of the images.

It is worth noting that the shown example covers a small portion of a whole Sentinel-1 image (around the 10\% of the image). However, the key point, is that it can be applied at a regional level in all the Shoutern Patagonia Ice field and in a systematic way each 12 days, providing a new source of knowledge about the glacier dynamics changes at both short and medium term.

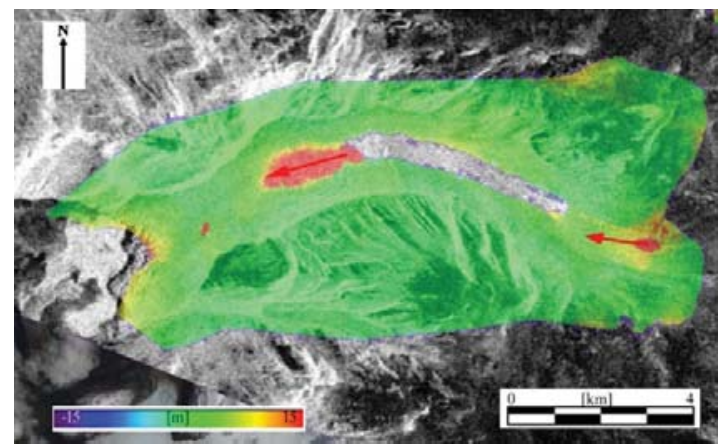

Figure 6. Amalia glacier displacement map.

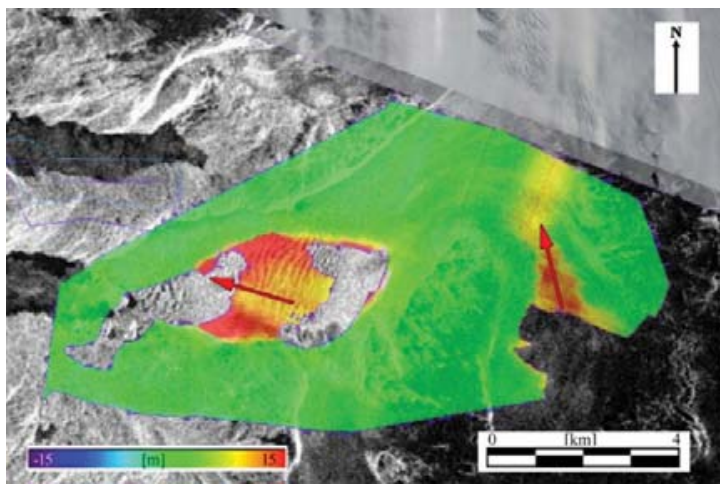

Figure 7. Asia glacier displacement map. 


\section{DISCUSION AND CONCLUSION}

In this work are shown two examples of application of Sentinel1 imagery for displacement monitoring in Patagonia region. Both examples are used to illustrate how well and consolidated techniques as SAR interferometry and offset tracking can be used with Sentinel-1 imagery to provide key information for displacement measurement and monitoring.

The example of Punta Arenas provides a displacement map that shows the main active areas in the Punta Arenas metropolitan area. Compared to old sensors, Sentinel-1 provides the capability to periodically update these types of maps providing not only inputs on terms of past information but also key inputs for urban planning, geohazard prevention and restoration activities.

The Shoutern Patagonia Icefield glacier example demonstrates the capabilities of using Sentinel-1 amplitude images to measure displacements. Even though this type of analysis provides lower precision compared to phase results, it is not affected by aliasing errors and therefore can measure very fast displacements (m/day). Moreover, taking into account that the Sentinel-1 precise orbits are good enough the achieve sub-pixel precision in the images co-registration, the offset tracking method provides absolute displacements which can be useful in situations where all the area is prone to displacements. Last, but not least, the acquisition policy, that assures one images each 12 days in that region, allows the systematic application of such approach providing key information about the glacier dynamics evolution.

\section{ACKNOWLEDGEMENTS (OPTIONAL)}

This work has been partially funded by the Ministry of education and professional training of the Spanish government through the program abroad stages for young researchers José Castillejo (Ref: CAS19/00190) and by the Spanish Ministry of Economy and Competitiveness through the DEMOS project "Deformation monitoring using Sentinel-1 data" (Ref: CGL2017-83704-P).

\section{REFERENCES}

Amelung, F., Galloway, D.L., Bell, J.W., Zebker, H.A., Laczniak, R.J., 1999. Sensing the ups and downs of Las Vegas: InSAR reveals structural control of land subsidence and aquifersystem deformation. Geology 27 (6), 483-486.

Antonielli, B., Monserrat, O., Bonini, M., Righini, G., Sani, F., Luzi, G., Feyzullayev, A. A., Aliyev, C.S., 2014. Pre-eruptive ground deformation of Azerbaijan mud volcanoes detected through satellite radar interferometry (DInSAR). Tectonophysics 637, 163-177.

Barra, A., Monserrat, O., Mazzanti, P., Esposito, C., Crosetto, M., \& Scarascia Mugnozza, G., 2016. First insights on the potential of Sentinel-1 for landslides detection. Geomatics, Natural Hazards and Risk, 7(6), 1874-1883.

Barra, A., Solari, L., Béjar-Pizarro, M., Monserrat, O., Bianchini, S., Herrera, G., ... \& Ligüerzana, S., 2017. A methodology to detect and update active deformation areas based on sentinel-1 SAR images. Remote Sensing, 9(10), 1002.

Biescas, E., Crosetto, M., Agudo, M., Monserrat, O., Crippa, B., 2007. Two radar interferometric approaches to monitor slow and fast land deformation. J. Surv. Eng. 133 (2), 66-71.
Bovenga, F., Wasowski, J., Nitti, D.O., Nutricato, R., Chiaradia, M.T., 2012. Using COSMO/SkyMed X-band and ENVISAT Cband SAR interferometry for landslides analysis. Remote Sens. Environ. 119, 272-285.

Brivio, P. A., Colombo, R., Maggi, M., \& Tomasoni, R., 2002. Integration of remote sensing data and GIS for accurate mapping of flooded areas. International Journal of Remote Sensing, 23(3), 429-441.

Burgess, E. W., Forster, R. R., \& Larsen, C. F., 2013. Flow velocities of Alaskan glaciers. Nature communications, 4, 2146.

Conesa, F., Devanthéry, N., Balbo, A., Madella, M., \& Monserrat, O., 2014. Use of satellite SAR for understanding long-term human occupation dynamics in the monsoonal semiarid plains of North Gujarat, India. Remote Sensing, 6(11), 11420-11443.

Crosetto, M., Monserrat, O., Cuevas, M., \& Crippa, B., 2011. Spaceborne differential SAR interferometry: Data analysis tools for deformation measurement. Remote Sensing, 3(2), 305-318.

Crosetto, M., Monserrat, O., Cuevas-González, M., Devanthéry, N., \& Crippa, B., 2016. Persistent scatterer interferometry: A review. ISPRS Journal of Photogrammetry and Remote Sensing, $115,78-89$.

Ferretti, A., Prati, C., Rocca, F., 2000. Nonlinear subsidence rate estimation using permanent scatterers in differential SAR interferometry. IEEE TGRS 38 (5), 2202-2212.

Ferretti, A., monti-guarnieri, A. V., Prati, C., Rocca, F., \& Massonnet, D. , 2007. INSAR Principles A. ESA publications.

Herrera, G., Davalillo, J.C., Mulas, J., Cooksley, G., Monserrat, O., Pancioli, V., 2009. Mapping and monitoring geomorphological processes in mountainous areas using PSI data: central Pyrenees case study. Nat. Hazards and Earth Syst. Sci. 9 (5), 1587-1598.

Hooper, A., Zebker, H., Segall, P., Kampes, B., 2004. A new method for measuring deformation on volcanoes and other natural terrains using InSAR persistent scatterers. Geophys. Res. Lett. 31 (23).

González, P. J., Bagnardi, M., Hooper, A. J., Larsen, Y., Marinkovic, P., Samsonov, S. V., \& Wright, T. J., 2015. The 2014-2015 eruption of Fogo volcano: Geodetic modeling of Sentinel-1 TOPS interferometry. Geophysical research letters, 42(21), 9239-9246.

Krieger, G., Moreira, A., Fiedler, H., Hajnsek, I., Werner, M., Younis, M., \& Zink, M., 2007. TanDEM-X: A satellite formation for high-resolution SAR interferometry. IEEE Transactions on Geoscience and Remote Sensing, 45(11), 3317-3341.

Lanari, R., Mora, O., Manunta, M., Mallorquí, J.J., Berardino, P., Sansosti, E., 2004. A small-baseline approach for investigating deformations on full-resolution differential SAR interferograms. IEEE TGRS 42 (7), 1377-1386.

Lundgren, P., Casu, F., Manzo, M., Pepe, A., Berardino, P., Sansosti, E., Lanari, R., 2004. Gravity and magma induced spreading of Mount Etna volcano revealed by satellite radar interferometry. Geophys. Res. Lett. 31 (4). 
Massonnet, D., Feigl, K.L., 1998. Radar interferometry and its application to changes in the Earth's surface. Rev. Geophys. 36 (4), 441-500

Mondini, A. C., Santangelo, M., Rocchetti, M., Rossetto, E., Manconi, A., \& Monserrat, O., 2019. Sentinel-1 SAR Amplitude Imagery for Rapid Landslide Detection. Remote Sensing, 11(7), 760 .

Monserrat, O., Moya, J., Luzi, G., Crosetto, M., Gili, J. A., \& Corominas, J., 2013. Non-interferometric GB-SAR measurement: application to the Vallcebre landslide (eastern Pyrenees, Spain). Natural Hazards and Earth System Sciences, 13(7), 1873.

Moran, M. S., Hymer, D. C., Qi, J., \& Sano, E., 2000. Soil moisture evaluation using multi-temporal synthetic aperture radar (SAR) in semiarid rangeland. Agricultural and Forest meteorology, 105(1-3), 69-80.

Osmanoğlu, B., Dixon, T. H., Wdowinski, S., Cabral-Cano, E., \& Jiang, Y., 2011. Mexico City subsidence observed with persistent scatterer InSAR. International Journal of Applied Earth Observation and Geoinformation, 13(1), 1-12.

Pettinato, S., Santi, E., Brogioni, M., Paloscia, S., Palchetti, E., \& Xiong, C., 2012. The potential of COSMO-SkyMed SAR images in monitoring snow cover characteristics. IEEE Geoscience and Remote Sensing Letters, 10(1), 9-13.

Raspini, F., Bianchini, S., Ciampalini, A., Del Soldato, M., Solari, L., Novali, F., ... \& Casagli, N., 2018. Continuous, semiautomatic monitoring of ground deformation using Sentinel-1 satellites. Scientific reports, 8(1), 7253 .

Singleton, A., Li, Z., Hoey, T., \& Muller, J. P., 2014. Evaluating sub-pixel offset techniques as an alternative to D-InSAR for monitoring episodic landslide movements in vegetated terrain. Remote Sensing of Environment, 147, 133-144.

Strozzi, T., Luckman, A., Murray, T., Wegmüller, U., Werner, C.L., 2002. Glacier motion estimation using SAR offset-tracking procedures. IEEE TGRS 40 (11), 2384-2391.

Schwalbe, E., Kröhnert, M., Koschitzki, R., Johnson, E., Cárdenas, C., Maas, H. G., 2017. Determination of spatiotemporal velocity fields at grey glacier using terrestrial image sequences and optical satellite imagery. In 2017 First IEEE International Symposium of Geoscience and Remote Sensing (GRSS-CHILE) , pp. 1-6.

Torres, R., Snoeij, P., Geudtner, D., Bibby, D., Davidson, M., Attema, E., Potin, P., Rommen, B., Floury, N., Brown, M., Navas Travera, I., Deghaye, P., Duesmann, B., Rosich, B., Miranda, N., Bruno, C., L'Abbate, M., Croci, R., Pietropaolo, A., Huchlerc, M., Rostan, F., 2012. GMES Sentinel-1 mission. Remote Sensing of Environment, 120, 9-24.

Van Zyl, J. J., 2001. The Shuttle Radar Topography Mission (SRTM): a breakthrough in remote sensing of topography. Acta Astronautica, 48(5-12), 559-565.

Villarroel, C., Tamburini Beliveau, G., Forte, A., Monserrat, O., \& Morvillo, M., 2018. DInSAR for a regional inventory of active rock glaciers in the dry Andes Mountains of Argentina and Chile with Sentinel-1 data. Remote Sensing, 10(10), 1588.
Waske, B., \& Braun, M., 2009. Classifier ensembles for land cover mapping using multitemporal SAR imagery. ISPRS Journal of Photogrammetry and Remote Sensing, 64(5), 450457.

Zhang, L., Ding, X., Lu, Z., 2011. Ground settlement monitoring based on temporarily coherent points between two SAR acquisitions. ISPRS J. Photogramm. Remote Sens. 66 (1), 146152. 